\title{
Quench protection performance measurements in the first MQXF magnet models
}

\author{
E. Ravaioli, G. Ambrosio, H. Bajas, G. Chlachidze,A. Fernandez Navarro, P. Ferracin, S. Izquierdo Bermudez, \\ P. Joshi, J. Muratore, F. Rodriguez-Mateos, GL. Sabbi, S. Stoynev, E. Todesco, and A. Verweij
}

\begin{abstract}
CERN and US LARP (LHC Accelerator Research Program) are jointly developing $\mathrm{Nb}_{3} \mathrm{Sn}$ quadrupole magnets to be installed in the LHC for its upgrade to higher luminosity. These magnets' quench protection system will include a combination of quench heaters attached to the coil surfaces and CLIQ units electrically connected to the magnets. Different protection elements have been characterized separately and simultaneously by implementing them on two 1.2 meter long model quadrupole magnets, tested at FNAL and CERN, and one 4.0 meter long mirror magnet tested at BNL. After analyzing the test data, their performances have been positively evaluated. Furthermore, the electro-thermal transients occurring after a quench have been simulated with the LEDET software and the results compared to experimental results. The preferred quench protection system configuration relies on outer-layer heaters and CLIQ. This solution is based on electrically robust components, achieves an effective reduction of the coils hot-spot temperature after a quench, and offers increased redundancy against component failures.
\end{abstract}

Index Terms-accelerator magnet, circuit modeling, CLIQ, quench protection, superconducting coil.

\section{INTRODUCTION}

A CHIEVING the targets of the High Luminosity LHC project requires the replacement of the superconducting quadrupole magnet system adjacent to the two high-luminosity interaction regions, ATLAS and CMS [1], [2]. This system, often referred to as inner triplet, will be composed of four separate electrical circuits, each including six $150 \mathrm{~mm}$ aperture, two-layer, $12 \mathrm{~T}, \mathrm{Nb}_{3} \mathrm{Sn}$ quadrupole magnets (MQXF), four of which with a magnetic length of $4.2 \mathrm{~m}$ (MQXFA) and two of $7.15 \mathrm{~m}$ (MQXFB) [3]-[6]. The main parameters of these magnets are summarized in Table I [3], [6], [7], and its magnetic field map in one half pole is shown in Fig. 1a.

Protecting these magnets against the effects of a quench is challenging due to the high magnetic-energy density stored in the coils [8]-[12]. An effective and fast heating mechanism is required to transfer most of the coil to the normal state in a

Work supported by the US Department of Energy through the US LHC Accelerator Research Program (LARP) and NSFC (Contract No. 11427904).

E. Ravaioli and GL. Sabbi are with the Lawrence Berkeley National Laboratory, Berkeley, CA. (e-mail: ERavaioli@lbl.gov).

G. Ambrosio, G. Chlachidze, and S. Stoynev are with the Fermilab National Accelerator Laboratory, Batavia, IL.

H. Bajas, A. Fernandez Navarro, P. Ferracin, S. Izquierdo Bermudez, F. Rodriguez-Mateos, E. Todesco, and A. Verweij are with CERN, Geneva, CH.

P. Joshi and J. Muratore are with Brookhaven National Laboratory, Upton, NY.

Manuscript received August 29, 2017
TABLE I

Main Magnet and Conductor Parameters [3], [6], [7].

\begin{tabular}{|c|c|c|c|}
\hline Parameter & Unit & MQXFA & MQXFB \\
\hline Nominal current, $I_{\text {nom }}$ & A & \multicolumn{2}{|c|}{16471} \\
\hline Ultimate current, $I_{\mathrm{ult}}$ & A & \multicolumn{2}{|c|}{17800} \\
\hline Peak field in the conductor at $I_{\text {nom }}$ & $\mathrm{T}$ & \multicolumn{2}{|c|}{11.4} \\
\hline Operating temperature & $\mathrm{K}$ & \multicolumn{2}{|c|}{1.9} \\
\hline Magnetic length, $l_{\mathrm{m}}$ & $\mathrm{m}$ & 4.20 & 7.15 \\
\hline Differential inductance at $I_{\text {nom }}$ & $\mathrm{mH}$ & 34.4 & 58.6 \\
\hline Stored energy at $I_{\text {nom }}$ & MJ & 4.7 & 7.9 \\
\hline Number of turns per pole, outer layer & - & \multicolumn{2}{|c|}{28} \\
\hline Number of turns per pole, inner layer & - & \multicolumn{2}{|c|}{22} \\
\hline Superconductor composition & - & \multicolumn{2}{|c|}{ Ti-alloyed $\mathrm{Nb}_{3} \mathrm{Sn}$} \\
\hline Number of strands & - & \multicolumn{2}{|c|}{40} \\
\hline Strand diameter, $d_{s}$ & $\mathrm{~mm}$ & \multicolumn{2}{|c|}{0.85} \\
\hline Bare cable width & $\mathrm{mm}$ & \multicolumn{2}{|c|}{18.363} \\
\hline Bare cable thickness & $\mathrm{mm}$ & \multicolumn{2}{|c|}{1.594} \\
\hline Insulation thickness & $\mathrm{mm}$ & \multicolumn{2}{|c|}{0.145} \\
\hline Copper/non-Copper ratio & - & \multicolumn{2}{|c|}{1.15} \\
\hline Filament twist pitch, $l_{\mathrm{tp}, \mathrm{f}}$ & $\mathrm{mm}$ & \multicolumn{2}{|c|}{19} \\
\hline Strand twist pitch, $l_{\mathrm{tp}, \mathrm{s}}$ & $\mathrm{mm}$ & \multicolumn{2}{|c|}{109} \\
\hline RRR of the copper matrix & - & \multicolumn{2}{|c|}{200} \\
\hline
\end{tabular}
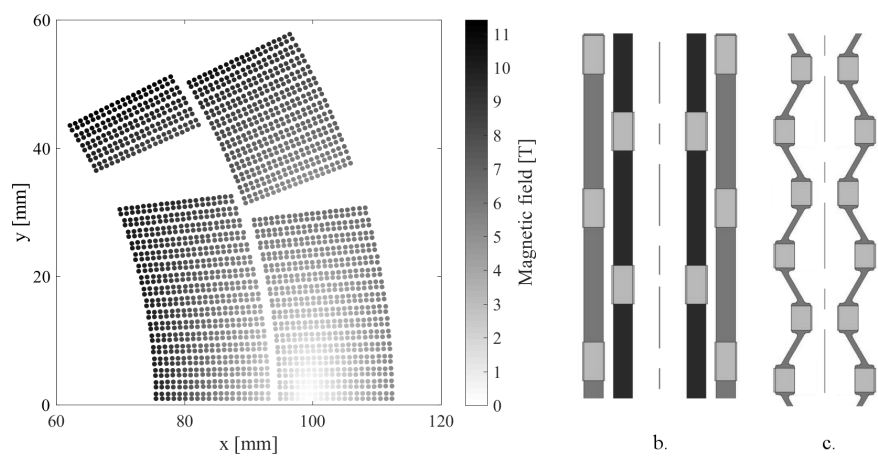

Fig. 1. High Luminosity LHC 12 T quadrupole magnet [8]. a. Cross-section of one half pole, showing the magnetic field at the nominal current of $16.47 \mathrm{kA}$. b. Heater strip traces attached to the coil's outer layer (OL-H). c. Heater strip traces attached to the coil's inner layer (IL-H).

few tens of millisecond, thus quickly discharging the magnet current and depositing the stored energy more uniformly in the winding pack. Furthermore, the quench protection system must depend on robust and reliable components to minimize the risk of a long LHC shut-down.

The inner triplet quench protection system relies on heaters and Coupling-Loss Induced Quench (CLIQ) technologies [13]-[15]. Heaters are $\mu \mathrm{m}$-thick stainless-steel strips glued to the coils' surfaces and separated from the conductor by a thin insulation layer. Upon quench detection, current is driven through them to heat them up and transfer energy to the coil via thermal diffusion. The designs of the 
TABLE II

MQXF HEATER PARAMETERS

\begin{tabular}{lcrrrr}
\hline \hline \multirow{2}{*}{ Parameter } & \multirow{2}{*}{ Unit } & \multicolumn{2}{c}{ MQXFA } & \multicolumn{2}{c}{ MQXFB } \\
& & OL-H & IL-H & OL-H & IL-H \\
\hline$N_{\mathrm{H}}$ & - & 2 & 2 & 2 & 2 \\
$l_{\mathrm{H}}$ & $\mathrm{m}$ & 4.2 & 4.2 & 7.0 & 7.0 \\
$N_{\mathrm{hs}}$ & - & 26 & 65 & 45 & 110 \\
$R_{\mathrm{hs}}$ & $\mathrm{m} \Omega$ & 27 & 44 & 27 & 44 \\
$R_{\mathrm{W}}$ & $\Omega$ & 0.6 & 0.6 & 0.6 & 0.6 \\
$R_{\mathrm{H}}$ & $\Omega$ & 2.9 & 4.2 & 4.6 & 6.7 \\
$U_{\mathrm{H}}$ & $\mathrm{V}$ & 570 & 565 & 900 & 900 \\
$C_{\mathrm{H}}$ & $\mathrm{mF}$ & 7.05 & 7.05 & 7.05 & 7.05 \\
$I_{\mathrm{H}}$ & $\mathrm{A}$ & 198 & 134 & 198 & 134 \\
$\tau_{\mathrm{H}}$ & $\mathrm{ms}$ & 20 & 30 & 32 & 47 \\
$P_{\mathrm{H}}$ & $\mathrm{Wcm}^{-2}$ & 213 & 98 & 213 & 98 \\
$E_{\mathrm{H}}$ & $\mathrm{Jcm}^{-2}$ & 2.16 & 1.45 & 3.42 & 2.32 \\
\hline \hline
\end{tabular}

heater strips attached to the MQXF coil's outer (OL-H) and inner layer (IL-H) are shown in Fig. 1b and 1c, respectively. CLIQ is a recently-developed method to heat up the coil by utilizing coupling loss. It relies on capacitive units that are connected to the coils to protect and discharged upon quench detection, hence introducing fast current changes in the coil sections. The resulting local magnetic-field change generates high inter-filament coupling loss [16], [17], which heats the copper matrix of the strands. The units are composed of simple, reliable, easy to repair electrical elements. CLIQ technology has been tested on magnets of different geometries, superconductor types, and sizes [15], [18]-[21], including full-scale accelerator magnets [22], [23].

In order to assess the performance of the quench protection system in conditions similar to the design targets, experimental campaigns have been carried out at the magnet test facilities of the Brookhaven Nation Laboratory (BNL) [24], European Organization for Nuclear Research (CERN) [25]-[27], and Fermi National Acceleration Laboratory (FNAL) [28]-[30].

Magnet discharges reproducing LHC relevant conditions were performed and compared to simulation results obtained with the LEDET (Lumped-Element Dynamic Electro-Thermal) program [13], [31], [32].

\section{Quench Protection System Elements}

The inner-triplet quench protection system includes heaters attached to the coils' surfaces and CLIQ units electrically connected to the magnets via dedicated current leads.

\section{A. Heaters}

Each heater circuit is composed of $N_{\mathrm{H}}=2$ strips in series connected to a unit including a capacitor bank of capacitance $C_{\mathrm{H}}[\mathrm{F}]$ charged to a voltage $U_{\mathrm{H}}[\mathrm{V}]$. The heater strip lengths $l_{\mathrm{H}}[\mathrm{m}]$, numbers and resistances of heating stations $N_{\mathrm{hs}}$ and $R_{\mathrm{hs}}[\Omega]$, and room-temperature wiring resistances $R_{\mathrm{w}}[\Omega]$ are reported in Table II, together with the resulting total heater circuit resistances $R_{\mathrm{H}}[\Omega]$, and peak currents $I_{\mathrm{H}}[\mathrm{A}]$. For each design, the peak power density deposited in the heating stations can be calculated as $P_{\mathrm{H}}=R_{\mathrm{hs}} I_{\mathrm{H}}^{2} / S_{\mathrm{hs}}\left[\mathrm{Wm}^{-2}\right]$, with $S_{\mathrm{hs}}\left[\mathrm{m}^{2}\right]$ the contact surface between one heating station
TABLE III

MQXF Heater-Delay Test Peak Power and Energy Density.

\begin{tabular}{lcccc}
\hline \hline Parameter & Unit & LF-OL-H & HF-OL-H & IL-H \\
\hline$P_{\mathrm{H}}$, MQXFS1 & $\mathrm{Wcm}^{-2}$ & 213 & 213 & 98 \\
$P_{\mathrm{H}}$, MQXFS3 & $\mathrm{Wcm}^{-2}$ & 123 & 123 & 122 \\
$P_{\mathrm{H}}$, MQXFPM1 & $\mathrm{Wcm}^{-2}$ & 179 & 192 & 83 \\
$E_{\mathrm{H}}$, MQXFS1 & $\mathrm{Jcm}^{-2}$ & 3.44 & 3.42 & 2.33 \\
$E_{\mathrm{H}}$, MQXFS3 & $\mathrm{Jcm}^{-2}$ & 2.60 & 2.60 & 2.59 \\
$E_{\mathrm{H}}$, MQXFPM1 & $\mathrm{Jcm}^{-2}$ & 2.75 & 2.85 & 1.88 \\
\hline \hline
\end{tabular}

and the coil. In first approximation, the heater current decays exponentially with time constant $\tau_{\mathrm{H}}=R_{\mathrm{H}} C_{\mathrm{H}}$ [s]. Hence, the total energy density deposited during decay can be calculated as $E_{\mathrm{H}} \approx P_{\mathrm{H}} \tau_{\mathrm{H}} / 2\left[\mathrm{Jm}^{-2}\right]$. The outer-layer heaters are designed to reach higher $P_{\mathrm{H}}$ and $E_{\mathrm{H}}$ than the inner-layer heaters, since they are attached to coil regions with lower magnetic field, and hence higher required energy to quench. The same type of heater unit is used to power MQXFA and MQXFB strips in order to reduce the number of required spare units. The units connected to MQXFA shorter, less resistive heater strips will be charged to a lower voltage to limit the peak heater current to the same value as MQXFB strips. As a consequence, the same $P_{\mathrm{H}}$ is achieved in the strips of both magnet types, but the time-constant and energy density deposited in MQXFA strips are smaller.

A key parameter for defining heater performance is the heater delay, defined as the time between the heater unit triggering and the onset of the induced coil transition to the normal state. Heater delays of selected heater strips are tested on two $1.2 \mathrm{~m}$ long quadrupole magnets (MQXFS1, MQXFS3) [25]-[29] and one $4.0 \mathrm{~m}$ long mirror magnet (MQXFPM1) [24]. The heater circuits parameters selected during the three test campaigns, reported in Table III, are set as similar as possible to the MQXFB design, compatibly with the hardware limitations in the magnet test facilities.

The delays measured after powering strips connected to the coils' outer layers are plotted in Fig. 2a. As expected, the delays decrease with the initial magnet current, since the conductor magnetic field increases and consequently the margin to quench decreases. For the same reason, delays after triggering the heaters attached to the high-field region of the coil (HF-OL-H) are shorter than after triggering low-field region heaters (LF-OL-H). At nominal current, delays measured in the three magnets are within 6 to $11 \mathrm{~ms}$ for HF-OL-H, and 11 to $19 \mathrm{~ms}$ for LF-OL-H. In the low to medium current range, MQXFS3 and MQXFPM1 low-field outer layer heater delays are shorter than expected [26].

The delays measured after triggering inner layer heaters are plotted in Fig. 2b. At nominal current, delays are in the 8 to $20 \mathrm{~ms}$ range.

For both outer and inner layer heaters, the consistency between the delays measured on short and long magnets confirms the good scalability of the heater technology with the magnet length.

Another key heater performance parameter is the minimum heater energy density required to initiate a transition to the normal state, $E_{\mathrm{H}, \min }\left[\mathrm{Jm}^{-2}\right]$. The results of $E_{\mathrm{H}, \text { min }}$ 

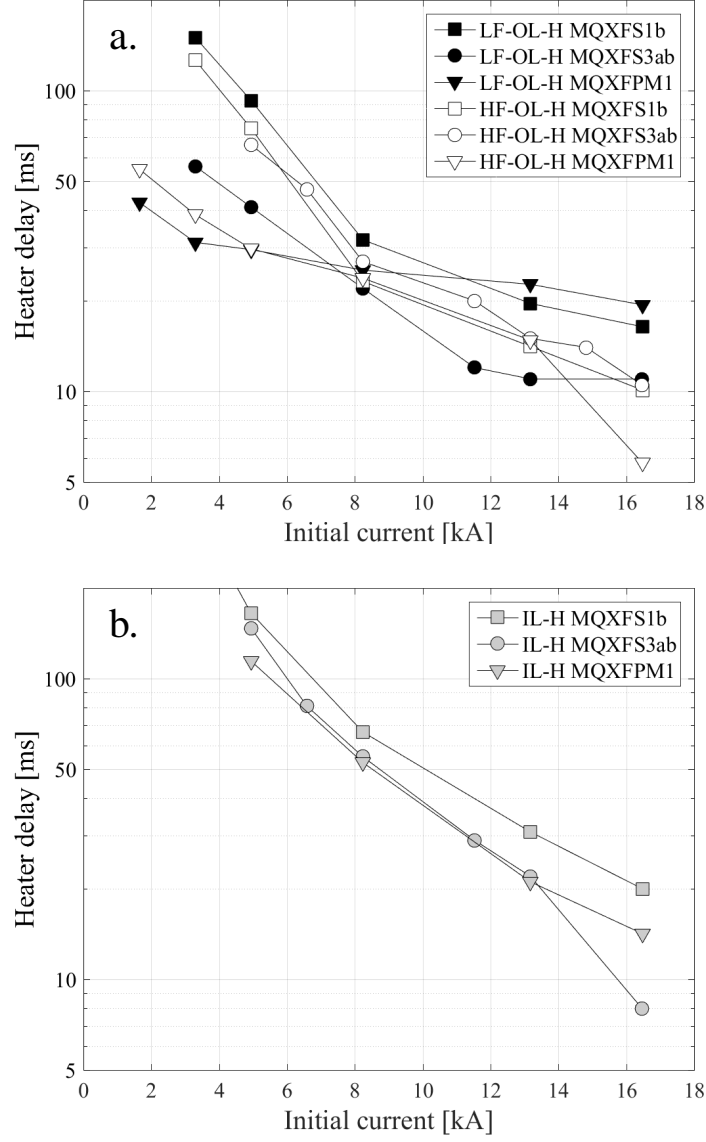

Fig. 2. Measured heater delay as a function of the magnet current [24]-[29]. Tests were performed with the parameters summarized in Table III. a. Heaters attached to the low- (LF-OL-H) and high-field (LF-OL-H) regions of the outer layer. b. Heaters attached to the inner layer (IL-H).

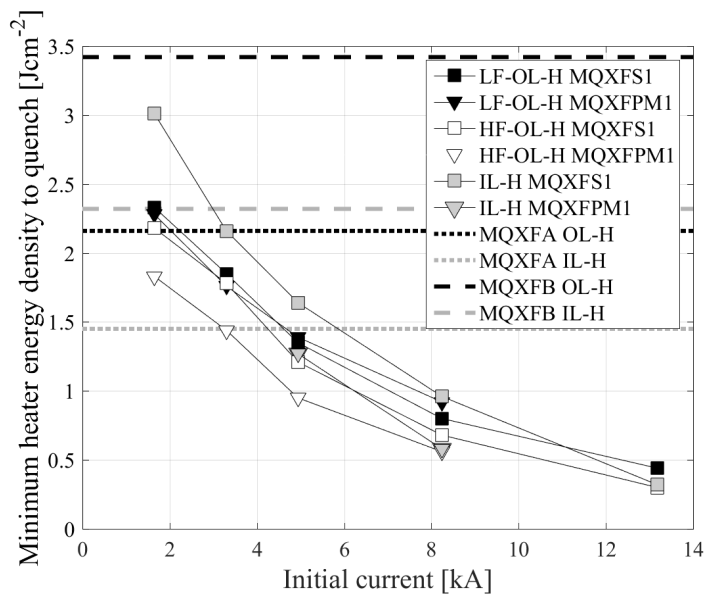

Fig. 3. Measured minimum heater energy density to quench as a function of the magnet current [24], [28], [29], compared to MQXFA and MQXFB design values. The capacitances of the heater units used during MQXFS1 and MQXFPM1 tests are 19.2 and $12.4 \mathrm{mF}$, respectively.

tests performed on the MQXFS1 and MQXFPM1 magnets are shown in Fig. 3. It is important to verify that the target deposited energies detailed in Table II are higher than $E_{\mathrm{H}, \min }$. This condition is satisfied at all tested current levels
TABLE IV

MQXF CLIQ PARAMETERS [8], [9].

\begin{tabular}{lccc}
\hline \hline Parameter & Unit & MQXFA & MQXFB \\
\hline CLIQ unit charging voltage & $\mathrm{V}$ & 1000 & 600 \\
CLIQ unit capacitance & $\mathrm{mF}$ & 40 & 40 \\
CLIQ circuit resistance & $\mathrm{m} \Omega$ & $\leq 100$ & $\leq 100$ \\
\hline \hline
\end{tabular}

by MQXFB targets for HF-OL-H and LF-OL-H. On the contrary, at $10 \% I_{\text {nom }}$ the MQXFA target is slightly lower than LF-OL-H $E_{\mathrm{H} \text {,min }}$. Moreover, the MQXFA and MQXFB target energy densities for IL-H are sufficient to initiate a quench only above about 35 and $20 \% I_{\text {nom }}$. However, since transferring a relatively small part of the coil to the normal state is sufficient to protect the magnet at low currents, the overall heater performance is adequate.

Heater long-term reliability is crucial for assuring magnet quench protection during the entire machine life-time, since multiple heater failures could significantly increase the peak magnet voltages to ground and hot-spot temperature [9]. During MQXFS1 and MQXFS3 test campaigns, several cases of heater electrical failures and heater-to-coil detachments occurred in the inner-layer heater strips, which question the implementation of this technology in the high luminosity LHC inner triplet magnets [26].

\section{B. Coupling-Loss Induced Quench System}

Each inner triplet circuit will include six $40 \mathrm{mF}$ CLIQ units for the protection of six magnets [8], [9]. To minimize the spare management effort, all units will be built following the same design, as detailed in Table IV. It has been shown that the peak power density deposited in the strands after triggering CLIQ is roughly proportional to the square of the ratio between the unit charging voltage $U_{0}[\mathrm{~V}]$ and the magnetic length $l_{\mathrm{m}}[\mathrm{m}]$ [13]. Thus, in order to target the same peak power density the units connected to the shorter magnets will be charged to a lower voltage.

In order to test CLIQ on the short model magnets in the same conditions as the design targets, a larger capacitance $C[\mathrm{~F}]$ is required. In fact, the CLIQ oscillation frequency is inversely proportional to $\sqrt{l_{\mathrm{m}} C}$ [13]. Due to hardware limitations the highest available CLIQ capacitance was $80 \mathrm{mF}$, and thus the oscillation frequency in the first model campaign was almost twice higher than the MQXF design target. As a result CLIQ performance was partly reduced, since the more frequent current polarity changes cause the coupling currents to reset more often, and hence decrease the coupling loss deposited over time.

\section{Quench Protection System Performance}

Quench protection system induced discharges have been performed on the first two model magnets to assess the performances of different protection configurations including heaters and CLIQ. The conductor parameters of the coils assembled in the MQXFS1 and MQXFS3 magnets are summarized in Table V. Note that two of the four MQXFS1 coils include OL-H strips featuring a different design [6], [33], 
TABLE V

Main Conductor Parameters of the Tested MQXF Short Model Coils [6], [7].

\begin{tabular}{lcccccc}
\hline \hline Coil & Assembly & Strand type & $\mathrm{Cu} /$ no-Cu ratio & $\mathrm{RRR}^{\mathrm{a}}$ & $l_{\mathrm{tp}, \mathrm{f}}[\mathrm{mm}]$ & $l_{\mathrm{tp}, \mathrm{s}}[\mathrm{mm}]$ \\
\hline Specifications & - & - & $1.2 \pm 0.1$ & $\geq 100$ & $19 \pm 3$ & $109 \pm 3$ \\
QXFS03 & MQXFS01 & RRP 108/127 & 1.165 & 250 & 14 & 109 \\
QXFS104 & MQXFS01 & RRP 132/169 & 1.174 & 105 & 19 & 109 \\
QXFS05 & MQXFS01 & RRP 108/127 & 1.182 & 255 & 14 & 109 \\
QXFS103 & MQXFS01 & RRP 132/169 & 1.174 & 135 & 19 & 109 \\
QXFS07 & MQXFS03 & RRP 108/127 & 1.199 & 170 & 14 & 109 \\
QXFS105 & MQXFS03 & RRP 132/169 & 1.218 & 140 & 19 & 109 \\
QXFS106 & MQXFS03 & RRP 132/169 & 1.218 & 140 & 19 & 109 \\
QXFS107 & MQXFS03 & RRP 132/169 & 1.214 & 140 & 19 & 109 \\
\hline \hline
\end{tabular}

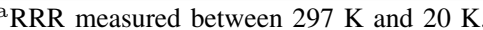

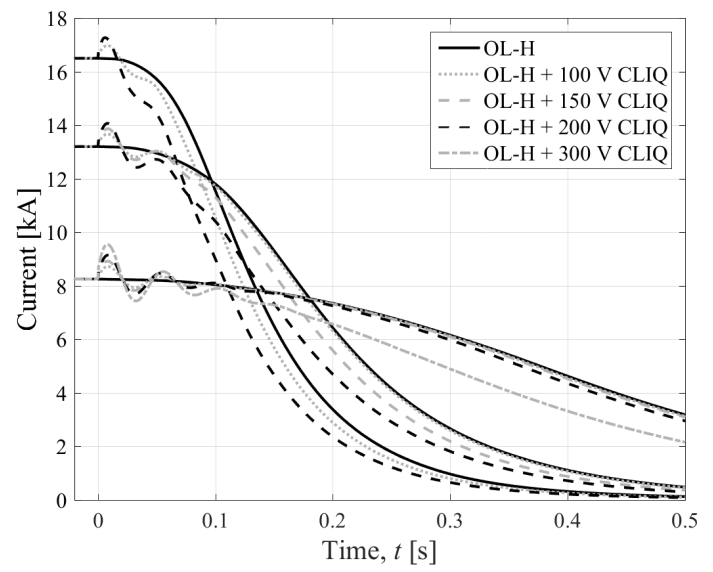

Fig. 4. Comparison between MQXFS1b magnet discharges obtained by triggering outer-layer heaters only, or outer-layer heaters and one CLIQ unit charged to different voltage levels. Measured magnet current $I_{\mathrm{m}}$ versus time.

and about half of the IL-H strips of both magnets experienced electrical problems and were not included in the protection scheme [26]. All heater and CLIQ parameters were selected to reproduce the MQXF target design performance as closely as allowed by hardware limitations. In particular, unit charging voltages were reduced by a factor roughly proportional to the ratio between the magnetic lengths of MQXFB and the model magnets, i.e. $\approx 7.15 / 1.19$.

\section{A. Experimental results}

Tests were performed on both magnets at different current levels by manually triggering the quench detection system, and hence activating the protection elements. Average triggering times for the heater and CLIQ units were 4 and $0.5 \mathrm{~ms}$, respectively. The currents measured during MQXFS1 magnet discharges for initial currents between 8.2 and $16.5 \mathrm{kA}$, corresponding to 50 to $100 \%$ of nominal current, are plotted in Fig. 4. Discharges obtained by simultaneously triggering outer-layer heaters and one CLIQ unit are compared to those obtained by triggering only OL-H. As expected, adding CLIQ to the protection system improves its performance. OL-H and CLIQ constitute an effective synergy since they are based on different heating mechanisms and deposit energy in different coil regions. In fact, OL-H heat up by thermal diffusion the outer layer, to which they are attached, whereas CLIQ heats up the strands where high magnetic-field changes are introduced, namely the inner layer and the mid-plane turns, utilizing inter-filament coupling loss [20], [21]. Similar discharges were performed for different CLIQ charging voltages to assess its impact on the magnet discharge. At nominal current, the curve obtained for $U_{0}=200 \mathrm{~V}$, which achieves a peak power deposited density similar to the MQXFB target, is significantly faster than that obtained with only OL-H.

A parameter often used to assess the protection system performance is the quench load, defined as the integral of the square of the magnet current during its discharge, $\int I_{\mathrm{m}}{ }^{2} \mathrm{dt}\left[\mathrm{A}^{2} \mathrm{~s}\right]$. Its value is roughly proportional to the energy deposited in the coil's hot-spot by joule heating. The quench loads obtained during MQXFS1 discharges in the range 10 to $108 \% I_{\text {nom }}$, calculated from the manual quench detection triggering, are shown in Fig. 5a. The current level at which CLIQ effectively reduces the quench load depends on the unit charging voltage. For a $200 \mathrm{~V}$ unit, achieving a peak power deposition similar to the MQXFB design target, the quench load is reduced for current levels above 50\% $I_{\text {nom }}$. At $I_{\text {nom }}$, the quench load is reduced by more than $20 \%$. For a $300 \mathrm{~V}$ unit, achieving a total energy deposition per unit volume similar to the MQXFB target, the quench load is reduced already at $30 \% I_{\text {nom }}$. Increasing the unit capacitance would improve the low-current performance. However, the protection in this current range is not critical and can be assured more cost-effectively by OL-H only. Therefore, it is important to assure the OL-H redundancy.

The quench loads obtained in similar tests performed on the MQXFS3 magnet are plotted in Fig. 5b. As one can calculate from the values reported in Table V, the MQXFS3 coil resistance is $1 \%$ and $10 \%$ higher than MQXFS1 at room temperature and $20 \mathrm{~K}$, respectively. Nonetheless, the quench loads measured during MQXFS3 OL-H tests are higher than MQXFS1. This indicates that MQXFS1 coil is transferred more quickly to the normal state. With respect to the OL-H discharges, a reduction of about $2 \mathrm{MA}^{2} \mathrm{~s}$ was achieved when both OL-H and IL-H were triggered simultaneously.

In the case of a real quench occurring in the magnet, the actual quench loads would be higher due to the additional time required to detect the quench on-start. At nominal current, this extra contribution is about $4 \mathrm{MA}^{2}$ s. In these conditions, the expected hot-spot temperature at the end of a discharge from 

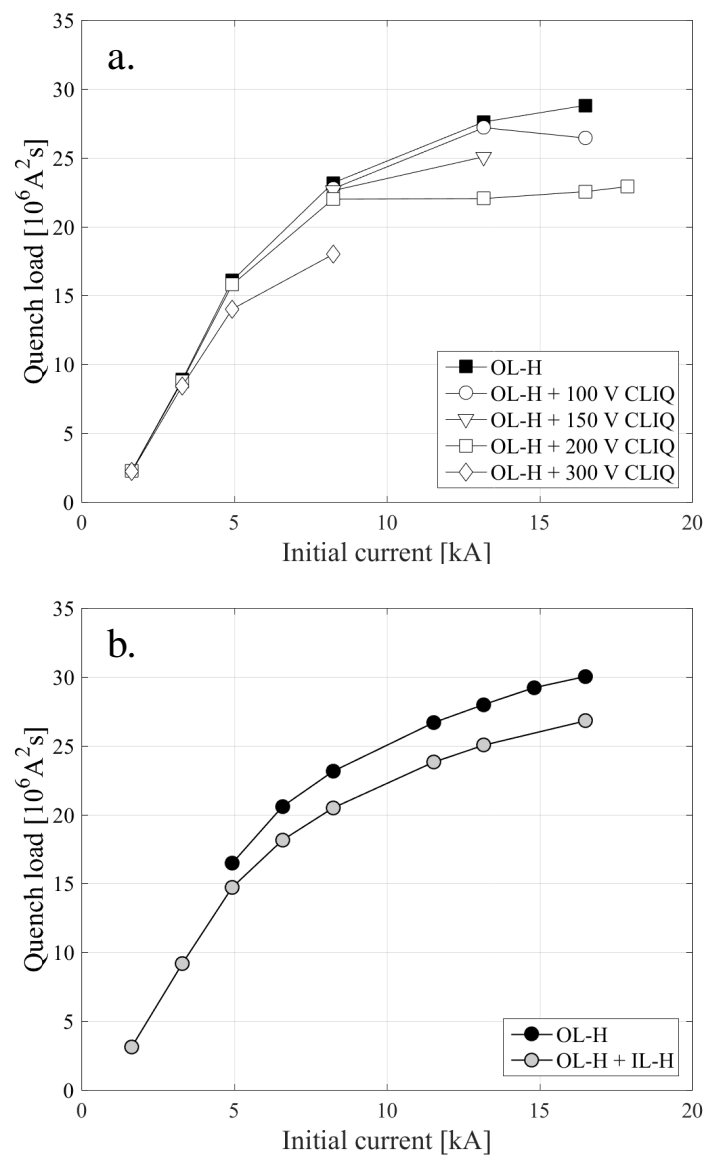

Fig. 5. Calculated quench loads as a function of the magnet current, obtained by triggering various combinations of heaters and CLIQ protection systems. a. MQXFS1 magnet [28], [29]. b. MQXFS3 magnet [25]-[27]. Values are calculated from the manual quench detection triggering. The values at currents below $5 \mathrm{kA}$ are further reduced due to the triggering of a $30 \mathrm{~m} \Omega$ energy-extraction system, occurring $1 \mathrm{~s}$ after quench detection.

$I_{\text {nom }}$ would be about $250 \mathrm{~K}$ for the protection configurations including OL-H together with either CLIQ or IL-H, and above $320 \mathrm{~K}$ for the OL-H case. Analyses showed that if strand parameter variability is high and/or failure cases occur the configuration including only OL-H is not sufficient to maintain the hot-spot temperature below the target limit of $350 \mathrm{~K}$ [9], which is considered a safe limit with respect to permanent degradation [34].

In conclusion, a protection strategy relying on heaters attached only to the coil's outer layer and on CLIQ appears the best choice in terms of hot-spot temperature reduction, redundancy improvement, and long-term system reliability.

\section{B. Simulation results}

The electro-magnetic and thermal transients occurring in the magnets during the tests are simulated with the LEDET program [13], [31], [32]. The comparison between the experimental and simulated quench loads after triggering the protection system at nominal current is shown in Table VI. Simulations overestimate the quench load by 2 to $12 \%$ for the heater-induced transients, and $18 \%$ for the OL-H and CLIQ discharge. Possible explanations for the quench load
TABLE VI

Quench Loads Calculated During MQXF Model Magnet Tests AT NOMINAL CURRENT AND COMPARISON WITH MODELING RESUlTS.

\begin{tabular}{|c|c|c|c|c|}
\hline \multirow{2}{*}{ Magnet } & \multirow{2}{*}{ Configuration } & \multicolumn{3}{|c|}{ Quench load $\left[10^{6} \mathrm{~A}^{2} \mathrm{~s}\right]$} \\
\hline & & Measurement & Model & Discrepancy \\
\hline MQXFS1 & OL-H & 28.8 & & $+12 \%$ \\
\hline MQXFS1 & OL-H + CLIQ & 22.5 & & $+18 \%$ \\
\hline MQXFS3 & OL-H & 29.2 & & $+7 \%$ \\
\hline MQXFS3 & OL-H + IL-H & 26.8 & & $+2 \%$ \\
\hline
\end{tabular}

overestimation are inaccuracies in the material properties, strand parameters, and model assumptions. Several effects might be implemented in the model in an attempt to improve its accuracy, including the resistance developed in the magnet ends, the strain-dependency of the $\mathrm{Nb}_{3} \mathrm{Sn}$ critical current, the effect of the strand twist-pitch on the ohmic loss per conductor unit length, and the temperature gradient within the conductor's metal, epoxy, and insulation volumes.

\section{CONCLUSION}

The quench protection system of the $\mathrm{Nb}_{3}$ quadrupole magnets for the high luminosity LHC upgrade was characterized by assessing the performance of its individual components in short and long magnet models. The protection scheme includes heater strips glued to the coil surfaces and CLIQ units electrically connected to the magnets.

The performances of individual heater strips in terms of delay between triggering and induced transition to the normal state are evaluated for the first two $1.2 \mathrm{~m}$ model magnets and one $4.0 \mathrm{~m}$ mirror magnet. The measured delays are sufficiently short to provide an effective quench heating. Scalability of the heater technology is successfully demonstrated after measuring similar delays for short and long heater strips. The long-term reliability of heater strips attached to the coil's inner layer is not demonstrated at this time since several cases of electrical failures and heater detachments occurred in the first two model magnets.

The addition of CLIQ units to the protection scheme allows achieving a faster magnet discharge, and hence reducing significantly the hot-spot temperature at the end of the quench transient. Tests performed by charging the CLIQ units at different voltages show that the selected design values are a correct compromise between reducing the hot-spot temperature and reducing the unit charging voltage.

The quench protection system configuration including outer-layer heaters and CLIQ is the preferred choice for its improved effectiveness, robustness, and redundancy. In fact, these two elements rely on separate electrical circuits, utilize distinct physical mechanisms, and deposit heat primarily in different coil locations.

\section{ACKNOWLEDGMENT}

The authors wish to thank Bernardo Bordini (CERN), Lance Cooley (FNAL), Dan Dietderich (LBNL), and Ian Pong (LBNL) for their help in tracing the information about MQXF superconductor parameters. 


\section{REFERENCES}

[1] G. Apollinari, O. Brüning, and L. Rossi, "High Luminosity LHC Project Description," CERN, Geneva, Tech. Rep. CERN-ACC-2014-0321, Dec 2014. [Online]. Available: https://cds.cern.ch/record/1974419

[2] G. Apollinari, I. Béjar Alonso, O. Brüning, M. Lamont, and L. Rossi, High-Luminosity Large Hadron Collider (HL-LHC): Preliminary Design Report. Geneva: CERN, 2015. [Online]. Available: http://cds.cern.ch/record/2116337

[3] P. Ferracin, G. Ambrosio, M. Anerella, F. Borgnolutti, R. Bossert, D. Cheng, D. R. Dietderich, H. Felice, A. Ghosh, A. Godeke, S. Izquierdo Bermudez, P. Fessia, S. Krave, M. Juchno, J. C. Perez, L. Oberli, G. Sabbi, E. Todesco, and M. Yu, "Magnet design of the $150 \mathrm{~mm}$ aperture low- $\beta$ quadrupoles for the High Luminosity LHC," IEEE Transactions on Applied Superconductivity, vol. 24, no. 3, pp 1-6, June 2014.

[4] G. Ambrosio, " $\mathrm{Nb}_{3} \mathrm{Sn}$ high field magnets for the High Luminosity LHC upgrade project," IEEE Transactions on Applied Superconductivity, vol. 25, no. 3, pp. 1-7, June 2015.

[5] E. Todesco, H. Allain, G. Ambrosio, F. Borgnolutti, F. Cerutti, D. Dietderich, L. Esposito, H. Felice, P. Ferracin, G. Sabbi, P. Wanderer, and R. V. Weelderen, "Design studies for the low-beta quadrupoles for the LHC luminosity upgrade," IEEE Transactions on Applied Superconductivity, vol. 23, no. 3, pp. 4002 405-4002 405, June 2013.

[6] G. Ambrosio, P. Ferracin, and al., "MQXFS1 Quadrupole Design Report,” Tech. Rep., 2016.

[7] L. D. Cooley, A. K. Ghosh, D. R. Dietderich, and I. Pong, "Conductor specification and validation for High-Luminosity LHC quadrupole magnets," IEEE Transactions on Applied Superconductivity, vol. 27, no. 4, pp. 1-5, June 2017.

[8] E. Ravaioli, G. Ambrosio, B. Auchmann, P. Ferracin, M. Maciejewski, F. Rodriguez-Mateos, G. Sabbi, E. Todesco, and A. Verweij, "Quench protection system optimization for the High Luminosity $\mathrm{LHC} \mathrm{Nb}_{3} \mathrm{Sn}$ quadrupoles," IEEE Transactions on Applied Superconductivity, vol. PP, no. 99 , pp. 1-1, 2017.

[9] E. Ravaioli, "Quench protection studies for the High-Luminosity LHC inner triplet circuit," CERN, Geneva, Tech. Rep. EDMS 1760496, 2016.

[10] G. Manfreda, G. Ambrosio, V. Marinozzi, T. Salmi, M. Sorbi, and G. Volpini, "Quench protection study of the $\mathrm{Nb}_{3} \mathrm{Sn}$ low- $\beta$ quadrupole for the LHC luminosity upgrade," IEEE Transactions on Applied Superconductivity, vol. 24, no. 3, pp. 1-5, June 2014.

[11] V. Marinozzi, G. Ambrosio, G. Bellomo, G. Chlachidze, H. Felice, M. Marchevsky, T. Salmi, M. Sorbi, and E. Todesco, "Study of quench protection for the $\mathrm{Nb}_{3} \mathrm{Sn}$ low- $\beta$ quadrupole for the LHC luminosity upgrade (HiLumi-LHC)," IEEE Transactions on Applied Superconductivity, vol. 25, no. 3, pp. 1-5, June 2015.

[12] E. Ravaioli, B. Auchmann, V. I. Datskov, J. Blomberg Ghini, K. Dahlerup-Petersen, A. M. Fernandez Navarro, G. Kirby, M. Maciejewski, F. Rodriguez-Mateos, H. H. J. ten Kate, and A. P. Verweij, "Advanced quench protection for the $\mathrm{Nb}_{3} \mathrm{Sn}$ quadrupoles for the High Luminosity LHC," IEEE Transactions on Applied Superconductivity, vol. 26, no. 3, pp. 1-6, April 2016.

[13] E. Ravaioli, "CLIQ," Ph.D. dissertation, Enschede, 2015, presented on 19 June 2015. [Online]. Available: http://doc.utwente.nl/96069/

[14] V. Datskov, G. Kirby, and E. Ravaioli, "AC-current induced quench protection system," Patent EP13 174 323.9, June 28, 2013.

[15] E. Ravaioli, V. I. Datskov, C. Giloux, G. Kirby, H. H. J. ten Kate, and A. P. Verweij, "New, Coupling Loss Induced, Quench protection system for superconducting accelerator magnets," IEEE Transactions on Applied Superconductivity, vol. 24, no. 3, pp. 1-5, June 2014.

[16] A. P. Verweij, "Electrodynamics of superconducting cables in accelerator magnets," Ph.D. dissertation, Twente U., Twente, 1995, presented on 15 Sep 1995. [Online]. Available: https://cds.cern.ch/record/292595

[17] M. Wilson, Superconducting Magnets, ser. Monographs on Cryogenics. Clarendon Press, 1983.

[18] E. Ravaioli, V. Datskov, A. Dudarev, G. Kirby, K. Sperin, H. ten Kate, and A. Verweij, "First experience with the new Coupling Loss Induced Quench system," Cryogenics, vol. 60, pp. 33-43, 2014. [Online]. Available: http://www.sciencedirect.com/science/article/pii/S0011227514000162

[19] E. Ravaioli, V. I. Datskov, G. Kirby, H. H. J. ten Kate, and A. P. Verweij, "A new hybrid protection system for high-field superconducting magnets," Superconductor Science and Technology, vol. 27, no. 4, p. 044023, 2014. [Online]. Available: http://stacks.iop.org/0953-2048/27/i=4/a=044023
[20] E. Ravaioli, H. Bajas, V. I. Datskov, V. Desbiolles, J. Feuvrier, G. Kirby, M. Maciejewski, G. Sabbi, H. H. J. ten Kate, and A. P. Verweij, "Protecting a full-scale $\mathrm{Nb}_{3} \mathrm{Sn}$ magnet with CLIQ, the new Coupling-Loss-Induced Quench system," IEEE Transactions on Applied Superconductivity, vol. 25, no. 3, pp. 1-5, June 2015.

[21] E. Ravaioli, V. I. Datskov, V. Desbiolles, J. Feuvrier, G. Kirby, M. Maciejewski, K. A. Sperin, H. H. ten Kate, A. P. Verweij, and G. Willering, "Towards an optimized Coupling-loss Induced Quench protection system (CLIQ) for quadrupole magnets," Physics Procedia, vol. 67, pp. 215-220, 2015. [Online]. Available: http://www.sciencedirect.com/science/article/pii/S1875389215004186

[22] E. Ravaioli, H. Bajas, V. I. Datskov, V. Desbiolles, J. Feuvrier, G. Kirby, M. Maciejewski, H. H. J. ten Kate, A. P. Verweij, and G. Willering, "First implementation of the CLIQ quench protection system on a full-scale accelerator quadrupole magnet," IEEE Transactions on Applied Superconductivity, vol. 26, no. 3, pp. 1-5, April 2016.

[23] E. Ravaioli, V. I. Datskov, G. Dib, A. M. Fernandez Navarro, G. Kirby, M. Maciejewski, H. H. J. ten Kate, A. P. Verweij, and G. Willering, "First implementation of the CLIQ quench protection system on a 14-m-long full-scale LHC dipole magnet," IEEE Transactions on Applied Superconductivity, vol. 26, no. 4, pp. 1-5, June 2016.

[24] J. M. et al., "Design and fabrication of the $1.9 \mathrm{k}$ magnet test facility at BNL, and test of the first $4 \mathrm{~m}$ long MQXF coil," IEEE Transactions on Applied Superconductivity.

[25] H. B. et al., "placeholder mqxfs3 test mt25 paper," IEEE Transactions on Applied Superconductivity.

[26] S. Izquierdo Bermudez, G. Ambrosio, H. Bajas, G. Chlachidze, J. F. Troitino, P. Ferracin, E. Ravaioli, S. Stoynev, E. Todesco, G. Sabbi, and G. Vallone, "Overview of the Quench Heater Performance for MQXF, the $\mathrm{Nb}_{3} \mathrm{Sn}$ low- $\beta$ Quadrupole for the High Luminosity LHC," IEEE Transactions on Applied Superconductivity.

[27] S. Izquierdo Bermudez, "Protection studies HCMQXFM001-CR000032," CERN, Geneva, Tech. Rep., 2017.

[28] G. Chlachidze, G. Ambrosio, M. Anerella, R. Bossert, E. Cavanna, D. W. Cheng, D. R. Dietderich, J. DiMarco, H. Felice, P. Ferracin, A. K. Ghosh, P. Grosclaude, M. Guinchard, A. R. Hafalia, E. F. Holik, S. Izquierdo Bermudez, S. T. Krave, M. Marchevsky, A. Nobrega, D. Orris, H. Pan, J. C. Perez, S. Prestemon, E. Ravaioli, G. Sabbi, T. Salmi, J. Schmalzle, S. E. Stoynev, T. Strauss, C. Sylvester, M. Tartaglia, E. Todesco, G. Vallone, G. Velev, P. Wanderer, X. Wang, and $\mathrm{M}$. Yu, "Performance of the first short model 150-mm-aperture $\mathrm{Nb}_{3} \mathrm{Sn}$ quadrupole MQXFS for the High-Luminosity LHC upgrade," IEEE Transactions on Applied Superconductivity, vol. 27, no. 4, pp. 1-5, June 2017.

[29] S. Stoyan and et al., "Summary of test results of MQXFS1 - the first short model $150 \mathrm{~mm}$ aperture $\mathrm{Nb}_{3} \mathrm{Sn}$ quadrupole for the high-luminosity lhc upgrade," IEEE Transactions on Applied Superconductivity.

[30] G. Ambrosio, G. Chlachidze, P. Wanderer, P. Ferracin, and G. Sabbi, "First Test Results of the $150 \mathrm{~mm}$ Aperture IR Quadrupole Models for the High Luminosity LHC," in 2nd North American Particle Accelerator Conference (NAPAC2016) Chicago, Illinois, USA, October 9-14, 2016, 2016. [Online]. Available: http://lss.fnal.gov/archive/2016/conf/fermilab-conf-16-440-td.pdf

[31] E. Ravaioli, B. Auchmann, M. Maciejewski, H. ten Kate, and A. Verweij, "Lumped-element dynamic electro-thermal model of a superconducting magnet," Cryogenics, pp. -, 2016. [Online]. Available: http://www.sciencedirect.com/science/article/pii/S0011227516300832

[32] E. Ravaioli, B. Auchmann, G. Chlachidze, M. Maciejewski, G. Sabbi, S. E. Stoynev, and A. Verweij, "Modeling of inter-filament coupling currents and their effect on magnet quench protection," IEEE Transactions on Applied Superconductivity, vol. 27, no. 4, pp. 1-8, June 2017.

[33] G. Ambrosio and al., "MQXFS1 Quadrupole Fabrication Report," Tech. Rep., 2016.

[34] G. Ambrosio, "Maximum allowable temperature during quench in $\mathrm{Nb}_{3} \mathrm{Sn}$ accelerator magnets," in Proceedings, WAMSDO 2013 Workshop on Accelerator Magnet, Superconductor, Design and Optimization: CERN Geneva, Switzerland, 15-16 Jan 2013, 2013, pp. 43-46. [Online]. Available: https://inspirehep.net/record/1277941/files/arXiv:1401.3955.pdf 